\title{
Sicyos angulatus ameliorates atherosclerosis through downregulation of aortic inflammatory responses in apolipoprotein E-deficient mice
}

\author{
YONG-HOON KIM ${ }^{1,2}$, JUNG-RAN NOH ${ }^{1}$, JUNG HWAN HWANG ${ }^{1,2}$, \\ KYOUNG-SHIM KIM $^{1,2}$, DONG-HEE CHOI ${ }^{1}$, JIN-PYO AN ${ }^{3}$, WON-KEUN OH ${ }^{3}$ and CHUL-HO LEE ${ }^{1,2}$ \\ ${ }^{1}$ Laboratory Animal Resource Center, Korea Research Institute of Bioscience and Biotechnology, \\ Daejeon 34141; ${ }^{2}$ Department of Functional Genomics, University of Science and Technology, \\ Daejeon 34113; ${ }^{3}$ Korea Bioactive Natural Material Bank, Research Institute of Pharmaceutical Sciences, \\ College of Pharmacy, Seoul National University, Seoul 08826, Republic of Korea
}

Received April 28, 2017; Accepted October 6, 2017

DOI: 10.3892/etm.2017.5271

\begin{abstract}
Sicyos angulatus (SA), a summer annual vine originating from Northeastern USA, is a widely distributed noxious invasive plant. However, the clinical application of SA has not been investigated previously. The purpose of present study was to determine the effects of SA on atherosclerosis and its underlying mechanism. Atherosclerosis was induced by feeding apolipoprotein E-deficient $\left(\mathrm{apoE}^{-/-}\right)$mice with an atherogenic diet for 8 weeks. SA was administered daily by oral gavage during induction of atherosclerosis. $\mathrm{ApoE}^{-/-}$mice treated with SA demonstrated a significant reduction in atherosclerotic plaque area in the whole aorta and aortic sinus compared with vehicle-treated mice. The plasma lipid profiles, including triglyceride, total cholesterol, high-density lipoprotein and low-density lipoprotein, were not affected by SA administration. Of note, gene expression levels of proatherogenic cytokines including tumor necrosis factor $\alpha(\operatorname{Tn} f \alpha)$ and interleukin-6 (Il-6) were significantly decreased in the aorta of SA administered apoE $\mathrm{E}^{-/}$mice. In lipopolysaccharide-stimulated RAW 264.7 macrophage cells, SA also inhibited the induction Tnfa, Il- 6 and $I l-1 \beta$ in a dose-dependent manner. Furthermore, gene expression levels of endothelial cell adhesion molecules, including vascular cell adhesion protein 1 and intercellular adhesion molecule 1 were reduced in the aorta of apoE $\mathrm{E}^{-/}$mice treated with $\mathrm{SA}$, which was followed by diminished aortic infiltration of monocytes/macrophages. In conclusion, to the best of our knowledge, this is the first study
\end{abstract}

Correspondence to: Dr Chul-Ho Lee, Laboratory Animal Resource Center, Korea Research Institute of Bioscience and Biotechnology, 125 Gwahak-ro, Yuseong-gu, Daejeon 34141, Republic of Korea

E-mail: chullee@kribb.re.kr

Key words: Sicyos angulatus, atherosclerosis, apolipoprotein E-deficient mice, inflammatory response, macrophage to demonstrate that $\mathrm{SA}$ is able to suppress the development of atherosclerosis by inhibiting the aortic expression of proinflammatory factors in atherogenic diet-fed apoE $\mathrm{E}^{-/-}$mice. The present study may provide novel insights into the application of the environmentally problematic weed SA as a therapeutically effective natural product for preventing atherosclerosis.

\section{Introduction}

Atherosclerosis is the primary underlying cause of myocardial infarction, stroke, unstable angina and sudden cardiac death, which account for the leading cause of death in the world $(1,2)$. In westernized societies, it has been reported that atherosclerosis is responsible for $\sim 50 \%$ of all deaths and the incidence is continuing to rise (1). Epidemiological studies have revealed that several important environmental and genetic risk factors associated with development and progression of atherosclerosis $(3,4)$. However, progress in defining the cellular and molecular interactions involved in atherosclerosis outbreak has been hindered by the disease's etiological complexity $(3,4)$. Lifestyle modifications are the first-line treatment, while medicines are usually the next step in treating atherosclerosis (5). Nevertheless, the current medicine does not sufficiently ameliorate atherosclerosis progression in a number of susceptible individuals $(6,7)$. Therefore, it is important to develop novel therapeutic strategies for atherosclerosis.

Atherosclerosis occurs in the subendothelial space of medium-sized arteries at regions of disturbed blood flow, and is triggered by an interplay between endothelial dysfunction and subendothelial lipoprotein retention, particularly low-density lipoprotein (LDL) (8). While in the arterial intima formation, LDL particles can undergo oxidation, becoming a putative promoter of atherogenesis (9). Once oxidized, LDL particles can induce endothelial and smooth muscle cell activation, secretion of inflammatory mediators and expression of adhesion molecules, a sequence of steps that culminates inflammatory cells especially monocyte in subendothelial space (9). Recruited monocytes can enhance the oxidation of LDL particles, leading to a vicious local loop (10). Once in 
the intima, monocytes become tissue macrophages, which can avidly internalize oxidized-LDL via scavenger receptors (10). This process generates macrophages loaded with lipids, also known as foam cells, which are a prominent feature of atherosclerotic plaque (11). By capturing these lipid particles, intimal macrophages can promote local vascular damage through various secreted mediators, such as proinflammatory cytokines and chemokines (11). Over time, this process stimulates inflammatory response that can cause intimal destruction, arterial thrombosis, and end-organ ischemia (12).

Sicyos angulatus (SA) is a summer annual vine belonging to the gourd family Cucurbitaceae originated from north-eastern USA $(13,14)$. SA is widely distributed throughout both Europe and Asia, and has been recognized as a noxious invasive alien species $(13,15)$. SA is a problem weed because of its aggressive habit in some summer crops, such as maize, soybean and pumpkin (13). SA is also common along water-courses and in open spaces, where it suppresses native vegetation (16). However, up to date, none of study has reported the usefulness and the beneficial roles of noxious plant SA in medicine field.

In the present study, we evaluated the effects of SA on atherosclerosis using RAW 264.7 cells, a murine macrophage cell line, and apolipoprotein E-deficient $\left(\mathrm{apoE}^{-/}\right)$mice.

\section{Materials and methods}

Preparation of SA extract. SA was purchased from Gangwon Herbs (Gangwon, Korea) on August, 2015. The voucher specimen (SNU2015-0008) was identified by Professor Won-Keun Oh, and was deposited at the College of Pharmacy, Seoul National University (Seoul, Korea). The SA extract was prepared and supplied by the Korea Bioactive Natural Material Bank (Seoul, Korea). Briefly, the dried aerial parts of SA were extracted with $70 \%$ ethanol three times for $6 \mathrm{~h}$ at room temperature. After that, the $70 \%$ ethanol-soluble extract was filtered and exhaustively concentrated to produce a $70 \%$ ethanolic extract under reduced pressure. The yield of SA extraction is $11 \%$. This SA extract was suspended in $0.5 \%$ carboxymethyl cellulose (CMC) at a concentration of $50 \mathrm{mg} / \mathrm{ml}$ as a stock solution, and the working solution of SA was adjusted to the intended concentrations for use in the in vitro and in vivo experiments in the present study.

Animal experiments. Male 8-week-old apoE ${ }^{-/-}$mice were obtained from Jackson Laboratory (Bar Harbor, ME, USA). Prior to experiments, mice were acclimatized to a $12-\mathrm{h}$ light/dark cycle at $22 \pm 2^{\circ} \mathrm{C}$ for 2 weeks with unlimited food and water in a specific pathogen-free facility. The mice were randomly divided into three groups: Those fed i) an atherogenic diet (no. 102571; Dyets Inc., Bethlehem, PA, USA) plus $0.5 \%$ carboxymethyl cellulose as the vehicle group $(n=13)$; ii) an atherogenic diet plus $300 \mathrm{mg} / \mathrm{kg}$ of SA as the SA300 group ( $\mathrm{n}=4)$; and iii) an atherogenic diet plus $500 \mathrm{mg} / \mathrm{kg}$ of SA as the SA500 group $(n=12)$. For en face Oil-red O staining of aorta, 9, 4 or 7 mice were used in the vehicle, the SA300 or the SA500 group, respectively. For aortic total RNA extraction, 4 or 5 mice were used in the vehicle or the SA500 group, respectively. We used The dose of SA were determined by preliminary tests. SA was administered daily by oral gavage for 8 weeks and changes of body weight were measured each week. All mice were euthanized by $\mathrm{CO}_{2}$ asphyxiation. All animal experiments were approved by the Institutional Animal Use and Care Committee of the Korea Research Institute of Bioscience and Biotechnology and were performed in accordance with the Guide for the Care and Use of Laboratory Animals published by the US National Institutes of Health.

Cell culture and SA treatment. The RAW 264.7 cells, a murine macrophage cell line, were purchased from the American Type Cell Culture (ATCC; Manassas, VA, USA) and were cultured in Dulbecco's modified Eagle's medium (DMEM) containing $10 \%$ fetal bovine serum, $100 \mathrm{U} / \mathrm{ml}$ penicillin and $100 \mu \mathrm{g} / \mathrm{ml}$ streptomycin in a humidified atmosphere $\left(5 \% \mathrm{CO}_{2} / 95 \%\right.$ air $)$ at $37^{\circ} \mathrm{C}$. The cells were pre-treated with different concentrations of SA $(100,200$ or $300 \mu \mathrm{g} / \mathrm{ml})$ for $1 \mathrm{~h}$, and then stimulated with lipopolysaccharide (LPS, $1 \mu \mathrm{g} / \mathrm{ml}$; Sigma-Aldrich; Merck KGaA, Darmstadt, Germany) or vehicle for $24 \mathrm{~h}$. The doses of SA were determined by preliminary tests using various concentration without cell cytotoxicity to examine the anti-inflammatory effects.

Quantitative real-time polymerase chain reaction. The total RNA was isolated from whole aortas or RAW 264.7 cells using TRIzol reagent (Invitrogen Life Technologies, Carlsbad, CA, USA), and reverse transcribed using the iScript ${ }^{\mathrm{TM}} \mathrm{cDNA}$ Synthesis kit (Bio-Rad Laboratories, Inc., Hercules, CA, USA). The resulting cDNA was subjected to qPCR using the StepOnePlus $^{\text {TM }}$ Real-Time PCR System (Applied Biosystems, Foster City, CA, USA) with AccuPower ${ }^{\circledR} 2 \mathrm{X}$ Greenstar qPCR Master Mix (Bioneer, Daejeon, Korea) according to the manufacturers' instructions. Relative gene expression levels were analyzed using the $2^{-\Delta \Delta \mathrm{Ct}}$ method and normalized against the expression of $18 \mathrm{~S}$ rRNA. The primer sequences used in the experiments are listed in Table I.

Blood analysis. At the end of the experimental period, blood samples were collected from the retro-orbital venous plexus of mice. Plasma was prepared by centrifugation of blood at $10,000 \mathrm{x} \mathrm{g}$ for $10 \mathrm{~min}$ at $4^{\circ} \mathrm{C}$ and subsequently storing it at $-80^{\circ} \mathrm{C}$. Plasma alanine aminotransferase (ALT), aspartate aminotransferase (AST), blood urea nitrogen (BUN), creatine kinase (CK), triglyceride, total cholesterol (TC), high density lipoprotein cholesterol (HDL-C) and low density lipoprotein cholesterol (LDL-C) were determined with an automated blood chemistry analyzer (Hitachi 7150; Hitachi, Ltd., Tokyo, Japan).

Analysis of atherosclerotic lesion formation in the aorta of ApoE ${ }^{-/}$mice. The whole aortas were isolated and the adventitial tissue were removed. After fixation in $10 \%$ neutral buffered formalin, the whole aortas were longitudinally dissected, and pinned flat on a rubber plate. Lipid plaques in the whole aorta were stained with Oil-red O (Sigma-Aldrich; Merck KGaA), and en face images were captured using a digital camera (Canon, Tokyo, Japan). The whole aorta surface and stained plaque areas were analyzed by digital image analysis software (Image Inside; GS Media, Daejeon, Korea).

Analysis of atherosclerotic lesion formation in the aortic sinus of $\mathrm{ApoE}^{-/-}$mice. The aortic sinuses were isolated, and 
Table I. Sequences of PCR primers used in this study.

\begin{tabular}{|c|c|c|}
\hline Gene & GenBank accession number & Primer sequence \\
\hline $\operatorname{Tnf} \alpha$ & NM_013693.3 & $\begin{array}{l}\text { Forward: 5'-TGGCCTCCCTCTCATCAGTT-3' } \\
\text { Reverse: 5'-CCTCCACTTGGTGGTTTGCT-3' }\end{array}$ \\
\hline Il6 & NM_031168.2 & $\begin{array}{l}\text { Forward: 5'-TTCCATCCAGTTGCCTTCTTG-3' } \\
\text { Reverse: 5'-GGGAGTGGTATCCTCTGTGAAGTC-3' }\end{array}$ \\
\hline$I l 1 \beta$ & NM_008361.4 & $\begin{array}{l}\text { Forward: 5'-CTACAGGCTCCGAGATGAACAAC-3' } \\
\text { Reverse: 5'-TCCATTGAGGTGGAGAGCTTTC-3' }\end{array}$ \\
\hline $1 l 10$ & NM_010548.2 & $\begin{array}{l}\text { Forward: 5'-GGGTTGCCAAGCCTTATCG-3' } \\
\text { Reverse: 5'-TCTCACCCAGGGAATTCAAATG-3' }\end{array}$ \\
\hline Vcaml & NM_011693.3 & $\begin{array}{l}\text { Forward: 5'-TGACTCCATGGCCCTCACTT-3' } \\
\text { Reverse: 5'-CGTCCTCACCTTCGCGTTTA-3' }\end{array}$ \\
\hline Icam 1 & NM_010493.3 & $\begin{array}{l}\text { Forward: 5'-TCACCAGGAATGTGTACCTGACA-3' } \\
\text { Reverse: 5'-ATCACGAGGCCCACAATGAC-3' }\end{array}$ \\
\hline
\end{tabular}
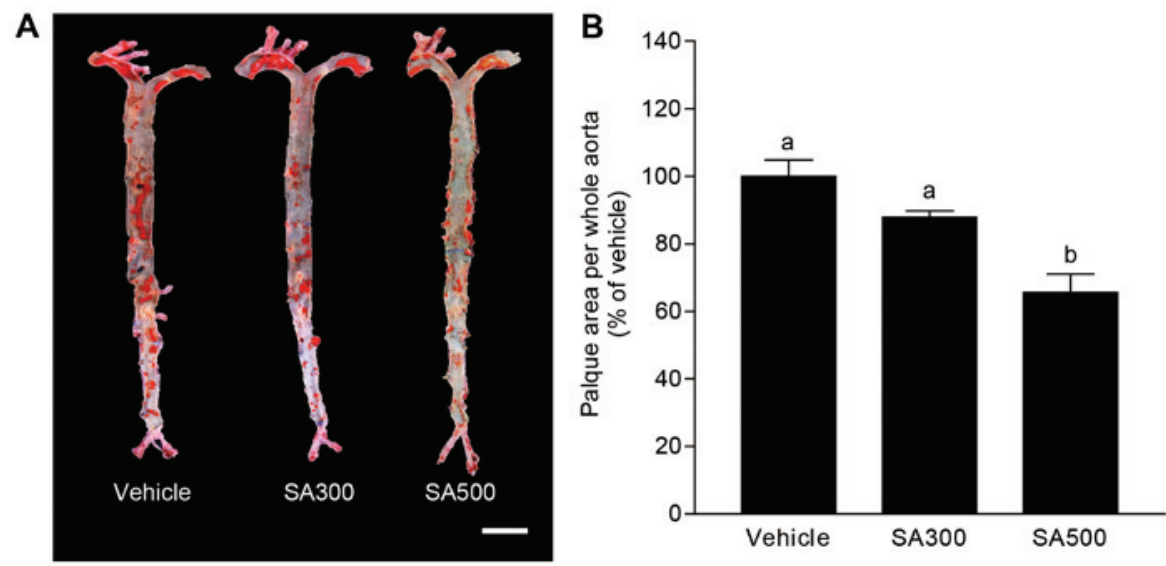

Figure 1. The effects of SA on development of atherosclerotic lesions in aorta of $\mathrm{apoE}^{-/}$mice. $\mathrm{ApoE}^{-/}$mice were fed an atherogenic diet either vehicle (vehicle group), 300 or $500 \mathrm{mg} / \mathrm{kg}$ of SA (SA300 or SA500 group) for 8 weeks, and whole aortas were excised. (A) Representative image for whole aorta of apoE ${ }^{-1-}$ mice $^{-1}$ in each group stained with Oil-red O. Scale bar, $500 \mu \mathrm{m}$. (B) Quantification of Oil-red O positive areas in whole aorta of apoE ${ }^{-/}$mice using image analysis software. Grouped quantitative data are presented as means \pm SEMs ( $n=4-9 /$ group). Significance was measured via Tukey-Kramer HSD test after the one-way ANOVA. ${ }^{\mathrm{a}, \mathrm{b}}$ Means are not sharing a common letter are significantly different among groups at $\mathrm{P}<0.05$.

fixed in $10 \%$ neutral buffered formalin. After fixation, the aortic sinuses were embedded in a Tissue-Tek optimal cutting temperature (OCT) compound (Sakura Finetek, Tokyo, Japan) and sectioned at a thickness of $8 \mu \mathrm{m}$ using a cryotome (Sakura Finetek). Cryostat sections of the aortic sinus were stained with Oil-red $\mathrm{O}$, and images were obtained using a light microscope (BX51; Olympus, Tokyo, Japan). The atheroma areas of the aortic sinus were quantified using digital image analysis software (image inside).

Analysis of monocyte/macrophage infiltration in the aortic sinus of $A p o E^{-/-}$mice. The infiltration of monocytes and macrophages were detected using anti-MOMA-2 antibody (Abcam, Cambridge, UK). The aortic sinuses were embedded in a Tissue-Tek OCT compound, and then sections of the aortic sinus ( $8 \mu \mathrm{m}$ thickness) were incubated with anti-MOMA-2 antibody (1:100), followed by Alexa Fluor $488 \mathrm{~nm}$ goat anti-rat IgG (1:200; Invitrogen Life Technologies) for visualization. Images of the aortic sinus were captured under confocal laser scanning microscopy (Carl Zeiss AG, Oberkochen, Germany).
Statistical analysis. Numerical data are presented as the mean \pm SEM. Comparisons two groups were performed using a two-tailed Student's t-test. Comparisons multiple groups were performed using Tukey-Kramer HSD test after the one-way ANOVA. The threshold of significance was set at $\mathrm{P}<0.05$.

\section{Results}

$S A$ reduces formation of atherosclerotic lesions in the aorta of apoE $E^{-/-}$mice. We examined the effect of SA on the formation of atherosclerotic lesions in vivo. SA was administered to $\mathrm{apoE}^{-/-}$mice under an atherogenic western diet for 8 weeks and the whole aorta was obtained. Fig. 1A shows aorta en face stained by Oil-red $\mathrm{O}$, and the area of atherosclerotic plaque was evaluated as shown in Fig. 1B. The percentage of aortic surface area covered by atherosclerotic lesions was tended to decrease in SA300 group $(87.9 \pm 1.3 \%)$ compared with vehicle group (Fig. 1). Interestingly, SA500 group (65.6 $\pm 2.4 \%)$ showed significantly reduced atherosclerotic lesion area in aorta en face compared with vehicle group (Fig. 1). These results could 
A

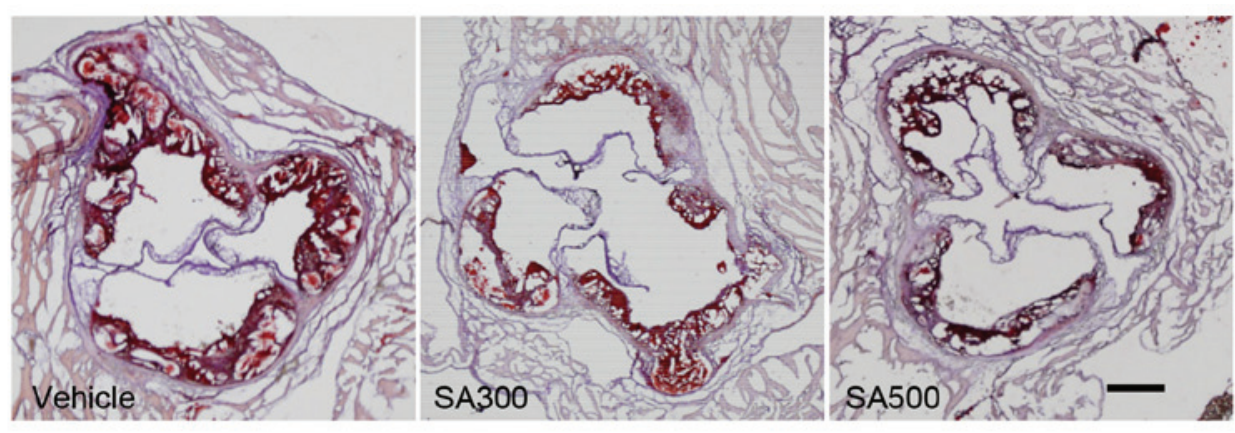

B

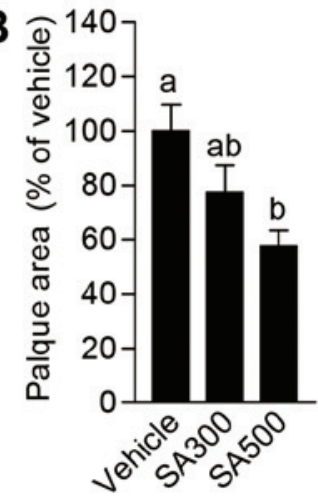

Figure 2. The effects of SA on formation of atherosclerotic plaques in aortic sinus of apoE ${ }^{-/}$mice. Animal experiments were performed as described in the legend of Fig. 1, and aortic sinus was excised. (A) Representative image for aortic sinus of apoE ${ }^{-/}$mice in each group stained with Oil-red O. Scale bar, $200 \mu \mathrm{m}$. (B) Quantification of atherogenic plaque areas in whole aorta of apoE ${ }^{-/}$mice using image analysis software. Grouped quantitative data are presented as means \pm SEMs ( $n=4-10$ /group). Significance was measured via Tukey-Kramer HSD test after the one-way ANOVA. ${ }^{a, b}$ Means are not sharing a common letter are significantly different among groups at $\mathrm{P}<0.05$.

Table II. Effects of SA on plasma biomarkers in ApoE KO mice.

\begin{tabular}{lccccllll}
\hline Groups & ALT (IU/l) & AST (IU/l) & BUN (mg/dl) & CK (IU/l) & TG (mg/dl) & TC (mg/dl) & HDL-C (mg/dl) & LDL-C (mg/dl) \\
\hline Vehicle & $17.8 \pm 1.2$ & $95.4 \pm 2.2$ & $28.8 \pm 0.4$ & $192.0 \pm 7.9$ & $120.0 \pm 1.4$ & $2819.2 \pm 25.4$ & $93.1 \pm 1.1$ & $2506.2 \pm 21.5$ \\
SA300 & $17.0 \pm 0.9$ & $85.5 \pm 2.1$ & $29.5 \pm 0.6$ & $159.5 \pm 8.5$ & $147.5 \pm 14.5$ & $2645.0 \pm 142.2$ & $100.0 \pm 6.1$ & $2302.5 \pm 143.5$ \\
SA500 & $13.7 \pm 0.6$ & $88.7 \pm 2.8$ & $29.5 \pm 0.3$ & $144.5 \pm 6.4$ & $129.2 \pm 2.5$ & $2778.3 \pm 33.2$ & $100.0 \pm 1.6$ & $2438.3 \pm 29.5$ \\
\hline
\end{tabular}

Grouped quantitative data are presented as mean \pm SEM (n=4-13/group). SA, Sicyos angulatus; ApoE KO, apolipoprotein E-deficient; ALT, alanine transaminase; AST, aspartate transaminase; BUN, blood urea nitrogen; TG, triglyceride; TC, total cholesterol; HDL-C, high-density lipoprotein cholesterol; LDL-C, low-density lipoprotein cholesterol.

suggest that SA has inhibitory effects on atherogenic lesion formation in $\mathrm{apoE}^{-/-}$mice.

SA diminishes atherosclerotic area in the aortic sinus of apoE $E^{-1}$ mice. To confirm the anti-atherogenic effects of SA in aorta, atherosclerotic lesions were analyzed in aortic sinus. Aortic sinus stained by Oil-red O (Fig. 2A), and the area of atherosclerotic lesions was analyzed (Fig. 2B). SA300 group $(77.4 \pm 6.6 \%)$ showed slightly reduced atherosclerotic lesion area in aortic sinus compared with vehicle group (Fig. 2). However, the percentage of aortic sinus covered by atherosclerotic plaque was significantly decreased in SA500 group $(57.6 \pm 2.3 \%)$ compared with vehicle group (Fig. 2). From these results, it is demonstrated that SA can ameliorate atherosclerosis in $\mathrm{apoE}^{-/}$mice.

Hyperlipidemia in apoE $E^{-1-}$ mice is not affected by SA administration. Hyperlipidemia is an elevation of plasma lipids, such as cholesterol or triglyceride, in the blood, and has been known as potential risk factor for atherosclerosis $(11,17)$. Therefore, we firstly evaluated whether the anti-atherogenic effects of SA is associated with plasma lipid modulation in atherogenic diet-fed $\mathrm{apoE}^{-/}$mice. Plasma triglyceride levels were not significantly different, and were tended to rather increase in both SA300 group and SA500 group compared with vehicle group (Table II). In addition, hypercholesterolemia shown by high plasma TC, HDL-C and LDL-C levels in vehicle group was comparable with both SA300 group and SA500 group (Table II). These results show that the SA may reduce atherogenic lesions independent of hyperlipidemia regulation in $\mathrm{apoE}^{-/ /}$mice.

SA inhibits the gene expression of proinflammatory cytokines in LPS-stimulated RAW 264.7 cells. The pathogenesis of atherosclerosis is inseparably related with proinflammatory cytokines released by various immune cells including macrophage $(18,19)$, and we identified whether SA can regulate proinflammatory reaction of immune cell in in vitro. RAW 264.7 cells, mouse macrophage cell line, were stimulated with LPS, and gene expression levels of proinflammatory cytokines were evaluated. LPS stimulation highly increased the expression levels of Tnf $\alpha$ (9.8 0.1 -fold), Il-6 (279.2 \pm 8.1 -fold) and $I l-1 \beta$ (39.6 \pm 0.8 -fold) compared with vehicle treated group. Intriguingly, co-treatment of SA eminently reduced all of these elevated proinflammatory cytokine expression levels in a dose dependent manner (Fig. 3). From these results, it is suggested that SA can diminish proinflammatory reaction in macrophage.

Proinflammatory cytokine expressions are reduced by $S A$ treatment in aorta of apoE $E^{-1-}$ mice. To examine anti-inflammatory effects of SA shown in in vitro, aortic expression levels of cytokines were measured in SA-treated $\mathrm{apoE}^{-/-}$mice. Because significant anti-atherogenic effects were only found in SA500 group, we focused on molecular biological changes in SA500 group. In accordance with in vitro results, 

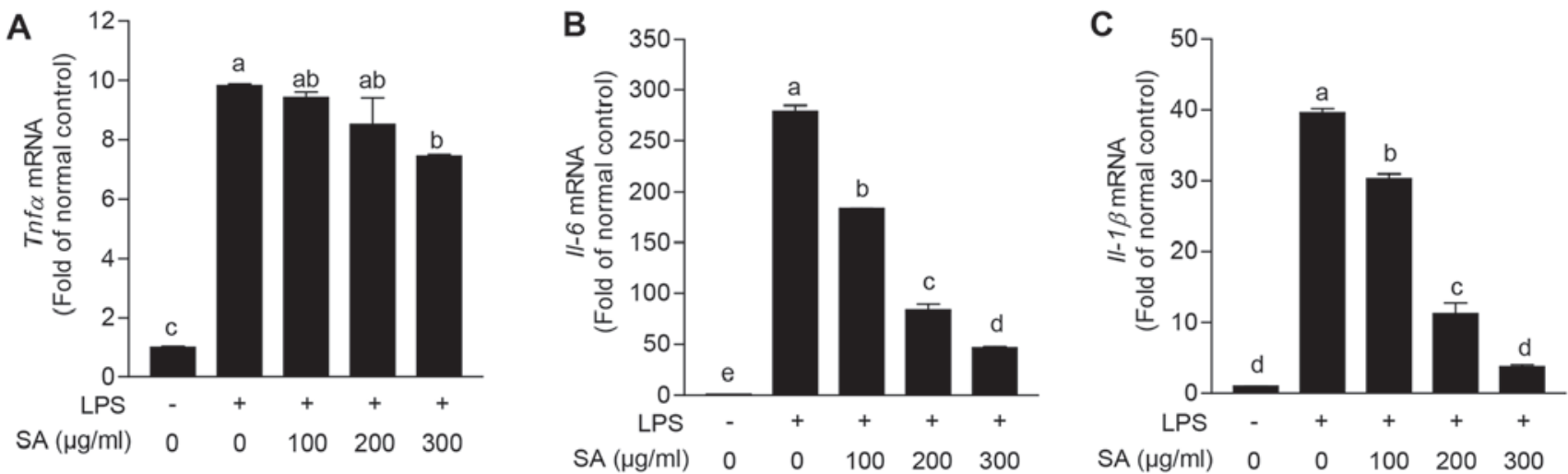

Figure 3. The effects of SA on gene expression of proinflammatory cytokines in lipopolysaccharide-stimulated RAW 204.7 cells. Gene expression levels of (A) Tnfa, (B) $I l-6$ and (C) $I l-1 \beta$ were analyzed by quantitative real-time PCR. Vehicle-only treated group was set to a value of 1 and average fold-change is shown. Grouped quantitative data are presented as means \pm SEMs from a minimum of three independent experiments. Significance was measured via Tukey-Kramer HSD test after the one-way ANOVA. ${ }^{\mathrm{a}-\mathrm{e}}$ Means are not sharing a common letter are significantly different among groups at $\mathrm{P}<0.05$.

A
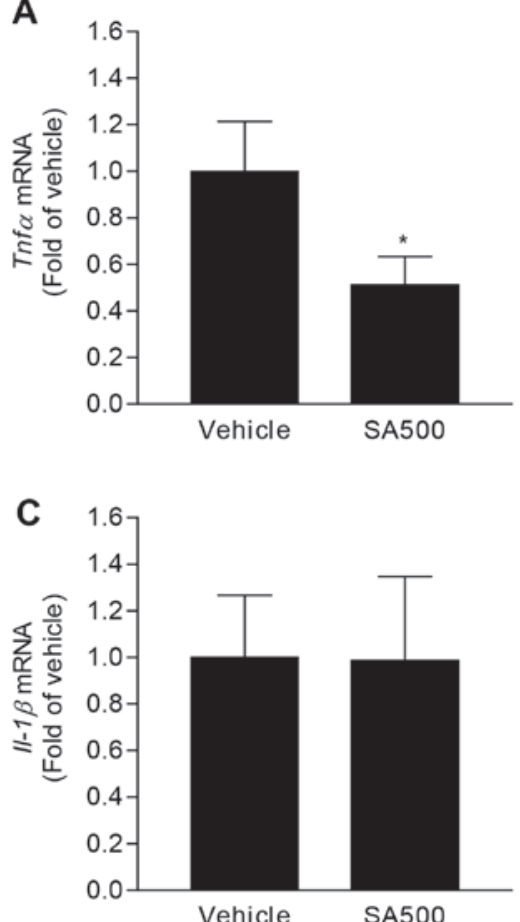

B
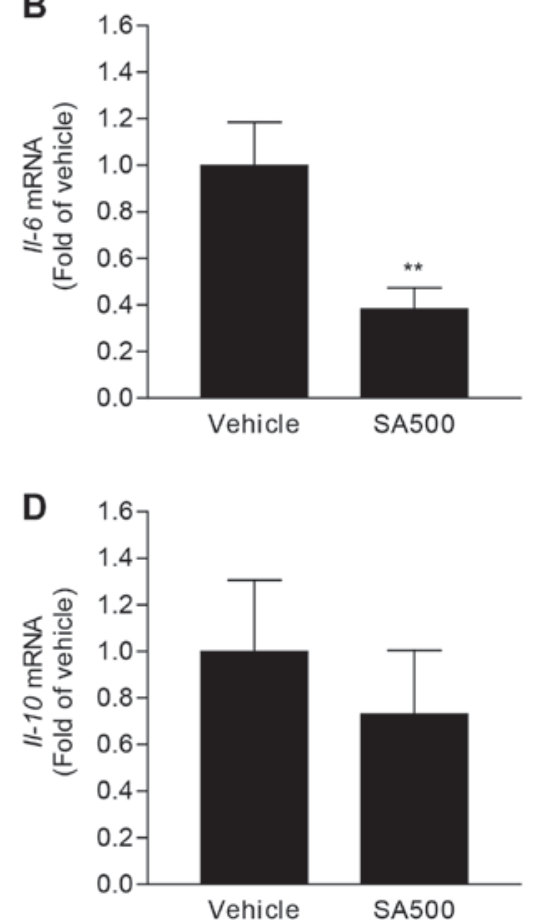

Figure 4. The effects of SA on gene expression of proinflammatory cytokines in aorta of apoE $\mathrm{E}^{-/-}$mice. ApoE $\mathrm{E}^{-/}$mice were fed an atherogenic diet either vehicle (vehicle group) or $500 \mathrm{mg} / \mathrm{kg}$ of SA (SA500 group) for 8 weeks, and whole aortas were excised. Gene expression levels of (A) Tnfa, (B) Il-6, (C) $\mathrm{Il}-1 \beta$ and (D) $\mathrm{Il-10}$ were analyzed by quantitative real-time PCR. Vehicle group was set to a value of 1 and average fold-change is shown. Grouped quantitative data are presented as means \pm SEMs ( $\mathrm{n}=4-5 /$ group). ${ }^{*} \mathrm{P}<0.05,{ }^{* *} \mathrm{P}<0.01$ (two-tailed Student's t-test).

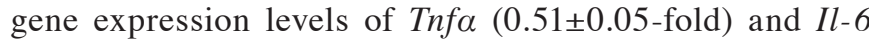
$(0.38 \pm 0.03$-fold $)$ were significantly decreased in aorta of SA500 group compared with vehicle group (Fig. 4A and B). However, Il-1 $\beta$ and Il-10 expression levels were comparable between vehicle group and SA500 group (Fig. 4C and D). These results would demonstrate that SA reduces proinflammatory responses in aorta of atherogenic diet-fed $\mathrm{apoE}^{-/-}$mice.

SA inhibits aortic expression of adhesion molecules and infiltration of monocyte/macrophage in apoE $E^{-/}$mice. We further evaluated aortic adhesion molecules, which is important for immune cell migration in pathogenesis of atherosclerosis (20). Interestingly, Vcam-1 expression level was significantly diminished in aorta of SA500 group $(0.31 \pm 0.03$-fold) compared with vehicle group (Fig. 5A) SA500 treatment also tended to reduce aortic Icam- 1 expression $(0.53 \pm 0.14$-fold) (Fig. 5A). In line with decreased aortic expression of adhesion molecules, expression of F4/80, a marker of murine macrophage, was significantly reduced in aorta of SA500 group $(0.37 \pm 0.04$-fold $)$ compared with vehicle group (Fig. 5B). Aortic Cd68 expression was also tended to decrease by SA administration in apoE $\mathrm{E}^{-/-}$mice (Fig. 5B). Based on aortic expression of macrophage marker, next, we confirmed macrophages infiltration using immunofluorescence staining for MOMA-2 in atherosclerotic plaque of aortic sinus (Fig. 5C). In accordance with aortic F4/80 and 

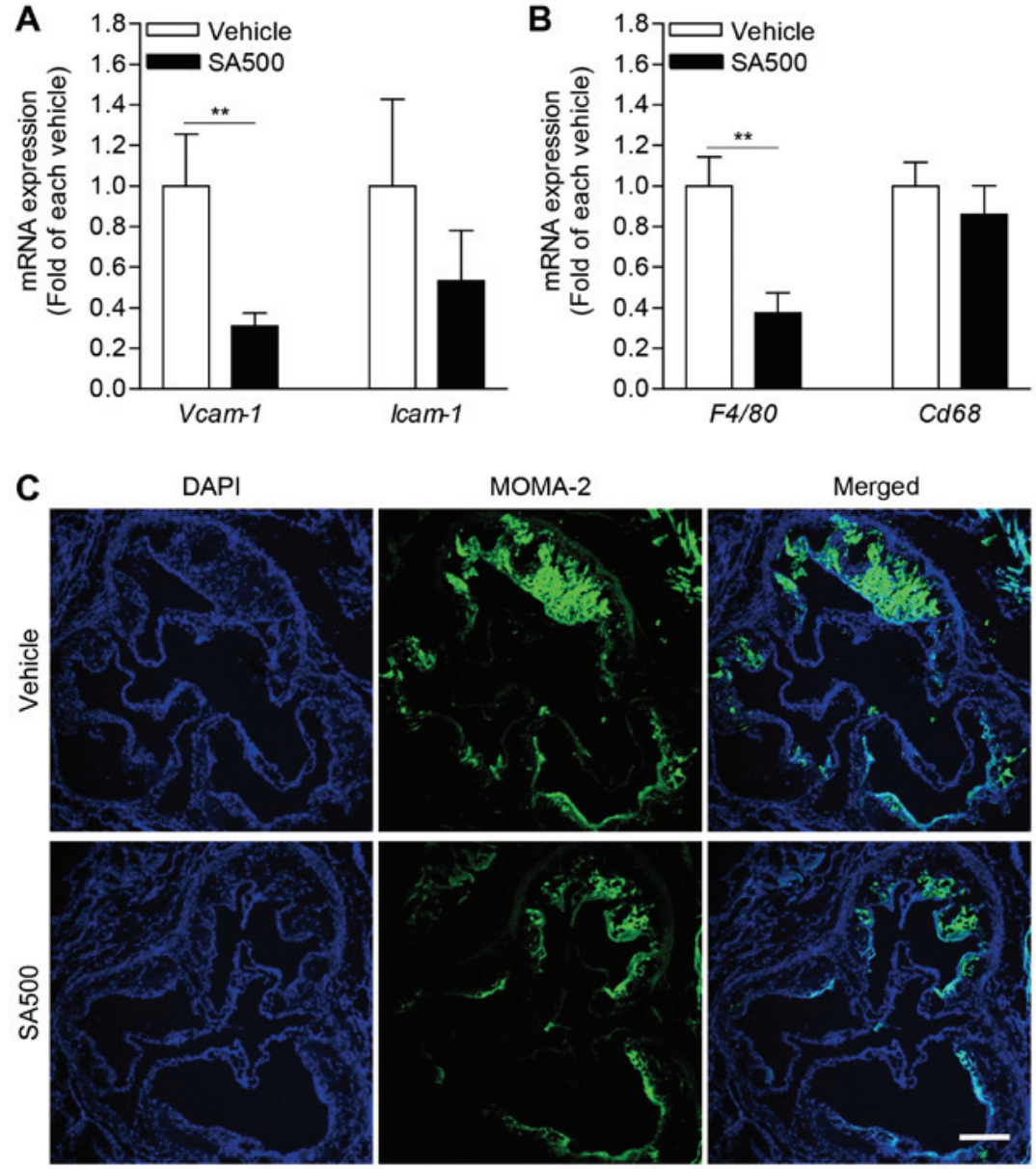

Figure 5. The effects of SA on aortic expression of adhesion molecules and infiltration of monocyte/macrophage in apoE ${ }^{-/}$mice. Animal experiments were performed as described in the legend of Fig. 4, and whole aortas and aortic sinus were excised. Gene expression levels of (A) adhesion molecules (Vcam- 1 and Icam-1) and (B) macrophage markers (F4/80 and Cd68) were analyzed by quantitative real-time PCR in whole aorta. Vehicle group was set to a value of 1 and average fold-change is shown. Grouped quantitative data are presented as means \pm SEMs ( $\mathrm{n}=4-5 /$ group). ${ }^{* *} \mathrm{P}<0.01$ (two-tailed Student's t-test). (C) Representative image for aortic sinus of apoE ${ }^{-/}$mice in each group stained with MOMA-2 antibody. Scale bar, $200 \mu \mathrm{m}$.

Cd68 expression levels, the areas stained by MOMA-2 were prominently diminished in SA500 group compared with vehicle group. Taken together, these findings suggest that SA treatment can inhibits aortic adhesion molecule expression followed by reduced monocyte/macrophage infiltration.

SA administration does not show toxicological phenotype in $a p o E^{-/}$mice. To evaluate the toxicity of SA in in vivo, toxicological markers found in blood were determined in plasma of 8-week-SA-treated apoE ${ }^{-/-}$mice. Plasma ALT, AST, BUN and CK levels were comparable among vehicle group, SA300 group and SA500 group (Table II). Moreover, the changes in body weight were not decreased but rather increased in both SA300 group and SA500 group compared with vehicle group (data not shown). Based on these results, it is suggested that long-term treatment of SA, at least for 8 weeks, does not evoke significant toxic effects in $\mathrm{apoE}^{-/-}$mice.

\section{Discussion}

The present study demonstrated a novel beneficial effects and useful roles of noxious plant SA in atherosclerosis, and provided the insight into anti-inflammatory potency of SA. When SA was administered to apo $\mathrm{E}^{-/-}$mice under an atherogenic western diet for 8 weeks, atherosclerosis was prominently ameliorated, as revealed by smaller atherosclerotic lesion area, reduced expression of proinflammatory cytokines and adhesion molecules, and decreased monocyte/macrophage infiltration in whole aorta or aortic sinus.

Atherosclerosis is a complex disease in which many processes contribute to lesion development (17). Until now, it is well accepted that hypercholesterolemia, especially high level of plasma LDL-C, play a key role in the initiation and progression of atherosclerosis $(11,17)$. In healthy conditions, the endothelium maintains the homeostasis of vascular wall via control of vascular tone (21). Nitric oxice (NO) plays a central atheroprotective role through the regulation of this vascular tone (22). Whereas, the exposure to high-LDL-C levels shows to decrease NO bioavailability, and causes endothelial dysfunction resulting in the preceding step to progression of atherosclerosis via LDL-C entry within the arterial intima (23). In clinical approach, lipid-lowering therapy with statins can lower LDL-C and effective in prevention of primary stroke by atherosclerosis (24). In contrary to LDL-C, HDL-C are generally inversely associated with the risk for the development of atherosclerosis (17). The major anti-atherosclerotic effect of HDL-C is reverse cholesterol transport, which scavenge cholesterol from the peripheral vasculature with transport to 
the liver where is it excreted in the biliary system $(17,25)$. In the present study, however, the levels of LDL-C, HDL-C and other lipids were not changed by SA administration in plasma of atherogenic diet-fed apoE ${ }^{-/}$mice. These results demonstrate that protective effect of SA is not related with modulation of plasma lipid profile in the context of atherogenesis.

Atherosclerosis is a well-known progressive chronic inflammatory disease, and many cytokines are expressed in atherosclerotic plaques $(10-12,18)$. Various cells involved in atherosclerosis are capable of producing cytokines and responding to them, and these cytokines can modulate atherogenesis $(18,26)$. Among cytokines, it has been demonstrated that TNF $\alpha$ plays a key role in development of atherosclerosis (27). TNF $\alpha$ cause reorganization of the actin and tubulin cytoskeletons in endothelial cells, thereby opening up gaps between adjacent cells (28). Moreover, TNF-deficient apoE $^{-/}$mice shows significantly smaller atherosclerotic lesion size in the aortic sinus than that of control mice (29). In accordance with these previous studies, anti-atherogenic effects of SA was correlated with reduced $T n f \alpha$ expression in aorta of atherogenic diet-fed mice and macrophage cells. Meanwhile, in the present study, SA also reduced expression of $\mathrm{Il}-6$ in aorta of apoE $\mathrm{E}^{-/-}$mice and macrophage cells. IL-6 has been suggested as proatherogenic cytokine, as evidenced by increased atherogenic lesion in IL-6 treated $a \mathrm{poE}^{-/-}$mice and destabilized plaques by letivirus-induced IL- 6 overexpression in mice $(30,31)$. Inhibition of IL-6 trans-signaling reduces atherosclerosis by decreasing endothelial cell activation and recruitment of monocytes (32). However, old IL-6-deficient apoE $E^{-/-}$and $\mathrm{LDLr}^{-/-}$mice show enhanced atherosclerotic plaque formation $(33,34)$. Although the role of IL-6 in atherogenesis still appears ambivalent, it could be considered that modulation of IL- 6 is related with regulatory roles of SA in atherosclerosis in apo $\mathrm{E}^{-/-}$mice. In regards to IL-1 $\beta$, mouse models of atherosclerosis have confirmed the proatherogenic properties of IL-1 $\beta$, associated with upregulation of endothelial adhesion molecules and activation of macrophages and vascular cells $(35,36)$. Bone marrow transplantation study in IL-1 receptor and apoE double knockout mice have shown that selective loss of IL-1 in the vessel wall reduces plaque burden rather than immune cells (37). Intriguingly, SA reduced $\mathrm{Il}-1 \beta$ expression not in aorta of apoE $\mathrm{E}^{-/}$mice but only in LPS-stimulated macrophage cells, and it could be supposed that anti-atherogenic effects of SA may not be dependent on IL-1 $\beta$. Collectively, SA may reduce atherosclerotic lesions through regulation of several proatherogenic cytokines in apoE $E^{-/-}$mice.

It has been well-known that recruitment of circulating immune cells, particularly monocytes, is crucial for initiation and progression of atherosclerosis $(38,39)$. A triggering event for this process is induction of proatherogenic factors including cytokines, which stimulate the overlying endothelial cells to produce adhesion molecules (1). In this regard, TNF-deficiency in apoE ${ }^{-/-}$mice shows decreased atherosclerotic lesion in the aortic sinus, which is associated with decreased expression of VCAM-1 and ICAM-1 (29). In line with theses previous studies, SA significantly reduced Tnfa expression followed by declined expression of Vcam-1 and Icam- 1 in aorta, and ameliorated atherosclerosis in atherogenic diet-fed apoE ${ }^{-/-}$mice. Moreover, decreased expression of adhesion molecules may be accompanied by reduced monocyte/macrophage infiltration in aortic sinus of atherogenic diet-fed apoE $\mathrm{E}^{-/-}$mice. These findings suggest that SA improve atherosclerosis, in part, by downregulation of adhesion molecule expressions.

Previous studies on chemical constituents from SA reported that some sterols and flavonoids were consisted in this plant $(40,41)$. In the present study, we have not found the key compound for the anti-atherogenic effects of SA, but we are going to try to isolate chemical constituents by activity-guided fractionations with in immune cell lines. As we isolate active constituents with this in vitro cell line system, the biological activities of the isolated compounds will be tested and confirmed with atherogenic diet-fed $\mathrm{apoE}^{-/-}$mice. Further studies are needed and will be continued for the confirmation of key compound for the anti-atherogenic property of SA.

In conclusion, this is the first study to demonstrate that SA suppresses the development of atherosclerosis by inhibiting the expression of proatherogenic factors including inflammatory cytokines and adhesion molecules, which is followed by reduction of atherogenic plaque formation and immune cell infiltration in aorta of apoE ${ }^{-/-}$mice. The present study provides new insight into the usefulness and the beneficial effects of noxious plant SA in medicine field, and identifies the potentiality of SA as a therapeutically effective novel natural product for preventing atherosclerosis.

\section{Acknowledgements}

We appreciate In-Bok Lee, Young-Keun Choi, Jung-Hyun Choi and Yun-Jeong Seo for technical assistance. This study was supported by a grant from the National Research Foundation of Korea (NRF) and the Korean government (MSIP) (2016R1A2A1A05004858), KRIBB Research Initiative Program of the Republic of Korea, and the Development of Platform Technology for Innovative Medical Measurements Program from Korea Research Institute of Standards and Science (KRISS-2017-GP2017-0020).

\section{References}

1. Lusis AJ: Atherosclerosis. Nature 407: 233-241, 2000.

2. Benjamin EJ, Blaha MJ, Chiuve SE, Cushman M, Das SR, Deo R, de Ferranti SD, Floyd J, Fornage M, Gillespie C, et al: Heart Disease and Stroke Statistics-2017 Update: A Report From the American Heart Association. Circulation 135: e146-e603, 2017.

3. Vargas JD, Manichaikul A, Wang XQ, Rich SS, Rotter JI, Post WS, Polak JF, Budoff MJ and Bluemke DA: Common genetic variants and subclinical atherosclerosis: The Multi-Ethnic Study of Atherosclerosis (MESA). Atherosclerosis 245: 230-236, 2016.

4. Hicken MT, Adar SD, Hajat A, Kershaw KN, Do DP, Barr RG, Kaufman JD and Diez Roux AV: Air pollution, cardiovascular outcomes and social disadvantage: The Multi-ethnic Study of Atherosclerosis. Epidemiology 27: 42-50, 2016.

5. Spring B, Moller AC, Colangelo LA, Siddique J, Roehrig M, Daviglus ML, Polak JF, Reis JP, Sidney S and Liu K: Healthy lifestyle change and subclinical atherosclerosis in young adults: Coronary Artery Risk Development in Young Adults (CARDIA) study. Circulation 130: 10-17, 2014.

6. Tian J, Gu X, Sun Y, Ban X, Xiao Y, Hu S and Yu B: Effect of statin therapy on the progression of coronary atherosclerosis. BMC Cardiovasc Disord 12: 70, 2012.

7. Hoffmann H, Frieler K, Schlattmann P, Hamm B and Dewey M: Influence of statin treatment on coronary atherosclerosis visualised using multidetector computed tomography. Eur Radiol 20: 2824-2833, 2010 
8. Tabas I, García-Cardeña G and Owens GK: Recent insights into the cellular biology of atherosclerosis. J Cell Biol 209: 13-22, 2015.

9. Witztum JL and Steinberg D: Role of oxidized low density lipoprotein in atherogenesis. J Clin Invest 88: 1785-1792, 1991.

10. Libby P: Inflammation in atherosclerosis. Nature 420: 868-874, 2002.

11. Rocha VZ and Libby P: Obesity, inflammation, and atherosclerosis. Nat Rev Cardiol 6: 399-409, 2009.

12. Hansson GK and Libby P: The immune response in atherosclerosis: A double-edged sword. Nat Rev Immunol 6: 508-519, 2006

13. Kobayashi H, Kurokawa S and Ikeda K: Dairyland populations of bur cucumber (Sicyos angulatus) as a possible seed source for riverbank populations along the Abukuma River, Japan. Weed Biol Manag 12: 147-155, 2012.

14. Lee SM, Radhakrishnan R, Kang SM, Kim JH, Lee IY, Moon BK, Yoon BW and Lee IJ: Phytotoxic mechanisms of bur cucumber seed extracts on lettuce with special reference to analysis of chloroplast proteins, phytohormones and nutritional elements. Ecotoxicol Environ Saf 122: 230-237, 2015.

15. Hulina N: New dangerous weed in Croatia: Sicyos angulatus L. (Cucurbitaceae). Poljopr Znan Smotra 61: 259-264, 1996.

16. Watanabe O, Kurokawa S, Sasaki H, Nishida T, Onoue T and Yoshimura Y: Geographic scale distribution and occurrence pattern of invasive weeds. Grassl Sci 48: 440-450, 2002.

17. Badimon L and Vilahur G: LDL-cholesterol versus HDL-cholesterol in the atherosclerotic plaque: Inflammatory resolution versus thrombotic chaos. Ann N Y Acad Sci 1254: 18-32, 2012.

18. Ait-Oufella H, Taleb S, Mallat Z and Tedgui A: Recent advances on the role of cytokines in atherosclerosis. Arterioscler Thromb Vasc Biol 31: 969-979, 2011.

19. Ramji DP and Davies TS: Cytokines in atherosclerosis: Key players in all stages of disease and promising therapeutic targets. Cytokine Growth Factor Rev 26: 673-685, 2015.

20. Blankenberg S, Barbaux S and Tiret L: Adhesion molecules and atherosclerosis. Atherosclerosis 170: 191-203, 2003.

21. Merritt WT: Nitric oxide: An important bioregulator. Transplant Proc 25: 2014-2016, 1993

22. Gimbrone MA Jr and García-Cardeña G: Endothelial cell dysfunction and the pathobiology of atherosclerosis. Circ Res 118: 620-636, 2016

23. Vidal F, Colomé C, Martínez-González J and Badimon L: Atherogenic concentrations of native low-density lipoproteins down-regulate nitric-oxide-synthase mRNA and protein levels in endothelial cells. Eur J Biochem 252: 378-384, 1998

24. Law MR, Wald NJ and Rudnicka AR: Quantifying effect of statins on low density lipoprotein cholesterol, ischaemic heart disease, and stroke: Systematic review and meta-analysis. BMJ 326: 1423, 2003

25. Trigatti BL, Krieger M and Rigotti A: Influence of the HDL receptor SR-BI on lipoprotein metabolism and atherosclerosis. Arterioscler Thromb Vasc Biol 23: 1732-1738, 2003.

26. McLaren JE, Michael DR, Ashlin TG and Ramji DP: Cytokines, macrophage lipid metabolism and foam cells: Implications for cardiovascular disease therapy. Prog Lipid Res 50: 331-347, 2011
27. McKellar GE, McCarey DW, Sattar N and McInnes IB: Role for TNF in atherosclerosis? Lessons from autoimmune disease. Nat Rev Cardiol 6: 410-417, 2009.

28. Pober JS and Sessa WC: Evolving functions of endothelial cells in inflammation. Nat Rev Immunol 7: 803-815, 2007.

29. Ohta H, Wada H, Niwa T, Kirii H, Iwamoto N, Fujii H, Saito K, Sekikawa K and Seishima M: Disruption of tumor necrosis factor-alpha gene diminishes the development of atherosclerosis in ApoE-deficient mice. Atherosclerosis 180: 11-17, 2005.

30. Huber SA, Sakkinen P, Conze D, Hardin N and Tracy R: Interleukin-6 exacerbates early atherosclerosis in mice. Arterioscler Thromb Vasc Biol 19: 2364-2367, 1999.

31. Zhang K, Huang XZ, Li XN, Feng M, Li L, Cai XJ, Zhang C, Liu XL, Zhang MX, Zhang Y, et al: Interleukin 6 destabilizes atherosclerotic plaques by downregulating prolyl-4-hydroxylase alpha1 via a mitogen-activated protein kinase and c-Jun pathway. Arch Biochem Biophys 528: 127-133, 2012.

32. Schuett H, Oestreich R, Waetzig GH, Annema W, Luchtefeld M, Hillmer A, Bavendiek U, von Felden J, Divchev D, Kempf T, et al: Transsignaling of interleukin- 6 crucially contributes to atherosclerosis in mice. Arterioscler Thromb Vasc Biol 32: 281-290, 2012.

33. Schieffer B, Selle T, Hilfiker A, Hilfiker-Kleiner D, Grote K, Tietge UJ, Trautwein C, Luchtefeld M, Schmittkamp C, Heeneman S, et al: Impact of interleukin-6 on plaque development and morphology in experimental atherosclerosis. Circulation 110: 3493-3500, 2004.

34. Song L and Schindler C: IL-6 and the acute phase response in murine atherosclerosis. Atherosclerosis 177: 43-51, 2004.

35. Kirii H, Niwa T, Yamada Y, Wada H, Saito K, Iwakura Y, Asano M, Moriwaki H and Seishima M: Lack of interleukin-1beta decreases the severity of atherosclerosis in ApoE-deficient mice. Arterioscler Thromb Vasc Biol 23: 656-660, 2003.

36. Clarke MC, Talib S, Figg NL and Bennett MR: Vascular smooth muscle cell apoptosis induces interleukin-1-directed inflammation: Effects of hyperlipidemia-mediated inhibition of phagocytosis. Circ Res 106: 363-372, 2010.

37. Shemesh S, Kamari Y, Shaish A, Olteanu S, Kandel-Kfir M, Almog T, Grosskopf I, Apte RN and Harats D: Interleukin-1 receptor type-1 in non-hematopoietic cells is the target for the pro-atherogenic effects of interleukin-1 in apoE-deficient mice. Atherosclerosis 222: 329-336, 2012.

38. Weber C, Zernecke A and Libby P: The multifaceted contributions of leukocyte subsets to atherosclerosis: Lessons from mouse models. Nat Rev Immunol 8: 802-815, 2008.

39. Moore KJ, Sheedy FJ and Fisher EA: Macrophages in atherosclerosis: A dynamic balance. Nat Rev Immunol 13: 709-721, 2013.

40. Akihisa $\mathrm{T}$, Tamura $\mathrm{T}$ and Matsumoto $\mathrm{T}$ : 24-Methylene-25-methyllathosterol: A sterol from Sicyos angulatus. Phytochemistry 26: 575-577, 1987.

41. Na CS, Lee YH, Murai Y, Iwashina T, Kim TW and Hong SH: Flavonol 3,7-diglycosides from the aerial parts of Sicyos angulatus (Cucurbitaceae) in Korea and Japan. Biochem Syst Ecol 48 235-237, 2013 